\title{
Magnetic resonance imaging changes in multifidus and psoas muscles in patients with lumbar spinal stenosis
}

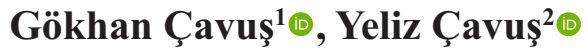 \\ ${ }^{1}$ Department of Neurosurgery, University of Health Sciences, Adana City Training and Research Hospital, Adana, Turkey \\ ${ }^{2}$ Department of Radiology, University of Health Sciences, Adana City Training and Research Hospital, Adana, Turkey
}

\section{ABSTRACT}

Objectives: The aim of this study is to evaluate the relationship between the spinal canal cross-sectional area (SCCSA) at the stenosis level, the cross-sectional areas of the multifidus (MF) muscle at the L3-S1 levels, and the cross-sectional areas of the psoas muscle (PSCSA) at the L3 level in patients with nerve compression due to lumbar spinal stenosis.

Methods: A total of 280 patients were included in the study, 140 in the control group and 140 with lumbar spinal stenosis that had been detected with magnetic resonance imaging (MRI). The patients' ages, gender, SCCSA at the level of stenosis, PSCSA at L3 level, MFCSA at L3-S1 levels, and degrees of atrophy caused by multifidus muscle fatty degeneration were evaluated and compared with the control group whether the comparison was statistically significant or not.

Results: PSCSA values at the L3 level and MFCSA at the L3-4 level ( $\mathrm{r}=0.555, p<0.01)$, MFCSA at the L4L5 level $(\mathrm{r}=0.559, p<0.01)$, and MFCSA at the L5-S1 level $(\mathrm{r}=0.429, \mathrm{p}<0.01)$ were found to have a statistically significant positive correlation. In addition, MFCSA at the L3-4 level $(p<0.001)$, MFCSA at the L4-5 level ( $p<0.001)$, MFCSA at the L5-S1 level $(p<0.001)$, the L3-L4 SCCSA $(p<0.001)$, and the L4-L5 SCCSA $(p<0.001)$ mean values were found to be statistically significantly lower than the mean of the cases in the control group.

Conclusions: Compared to the control group, a significant correlation was found with a decrease in the CSA of the multifidus muscle and the presence of fatty degeneration of the multifidus muscle in patients with spinal stenosis. However, in the CSA of the psoas muscle at the L3 level, it was found that there was no correlation between patients with spinal stenosis and the control group.

Keywords: fatty degeneration, stenosis, multifidus, spinal, psoas, cross-sectional area

L umbar spinal stenosis is the narrowing of the entrance and exit of the spinal canal, nerve root canal, and intervertebral foramen due to bone or soft tissue compression. As a result of disc degeneration, bone structures, and ligament hypertrophy, the spinal canal becomes narrow. If direct compression of the nerve root is involved in the physiopathology, it is ac- cepted that the lumbar muscles will also play a role in the clinical symptoms. Clinical symptoms of degenerative lumbar stenosis are usually seen in patients over 60 but may be seen at an earlier age in patients with developmental and acquired stenosis. The man/woman ratio in gender distribution is $2: 3$, but it usually manifests clinically with neurogenic claudica- 
tion or radicular symptoms [1].

The psoas muscle and the paraspinal muscles are important in maintaining the function of the lumbar spine. The multifidus muscle is the largest and most medial of the lumbar paraspinal muscles and is innervated by the medial branch of the segmental nerve root. The psoas muscle is innervated at the L1-L4 levels of the lumbar plexus by the branches of the anterior rami [2] Nerve root compression with spinal stenosis can cause morphological changes in the psoas and multifidus muscles [3].

Patients with clinical symptoms should be monitored by radiological studies to confirm that they have lumbar stenosis. MRI provides the most comprehensive imaging of the paraspinal muscle groups, psoas muscle, intervertebral discs, ligamentum flavum, spinal canal, and neuroforamen [4]. Computed tomography $(\mathrm{CT})$ scanning allows accurate evaluation of the bone structures including vertebral bodies and facet joints [5]. When evaluating the spine, an SCCSA below $145 \mathrm{~mm} 2$ at the level of stenosis is radiologically accepted as spinal stenosis [6].

Muscle morphology may be changed by muscle atrophy and fatty degeneration, both of which affect muscle performance. Many studies report using imaging methods such as MRI or CT scanning to evaluate this change $[7,8]$. Fat infiltration of the multifidus muscle on MRI was graded as "grade 0 " for $0-10 \%$ fat, "grade 1 " for $10-50 \%$ fat, and "grade 2 " for $>50 \%$ fat (Fig. 1) [9]. Apart from these studies, there are studies evaluating muscle cross-sectional areas [10, 11]. Farshad et al. [3] measured the distance of the multifidus muscle to the lamina in addition to the cross-sectional area. Yagi et al. [12] reported that trunk muscles play an important role in the spinal structure and that paraspinal muscle degeneration is associated with spinal deformity based on the evaluation of the cross-sectional area. However, there has been no study in which the cross-sectional areas of the psoas and multifidus muscles were evaluated together in patients with lumbar spinal stenosis.

The aim of this study is to demonstrate the crosssectional changes in the multifidus and psoas muscles in patients with lumbar spinal stenosis and those in the control group and to compare the degree of fatty degeneration in the multifidus muscles at the spinal stenosis level using MRI to evaluate whether the results are statistically correlated.

\section{METHODS}

A total of 280 patients were included in the study, 140 patients in the control group and 140 patients with lumbar spinal stenosis as detected with MRI in the radiology clinic between 01/01/2018-12/25/2020. Patients with previous spinal surgery, spondylolisthesis, spinal mass, vertebral fracture, or scoliosis were not included in the study. The control group patients were selected to correlate with the age and gender of the patient group. In the study, there were 54 men in both the patient and control group (38.6\%), and 86 women in the patient and control group (61.4\%). The mean age of the patient group was $58.2 \pm 8.34$ years and that

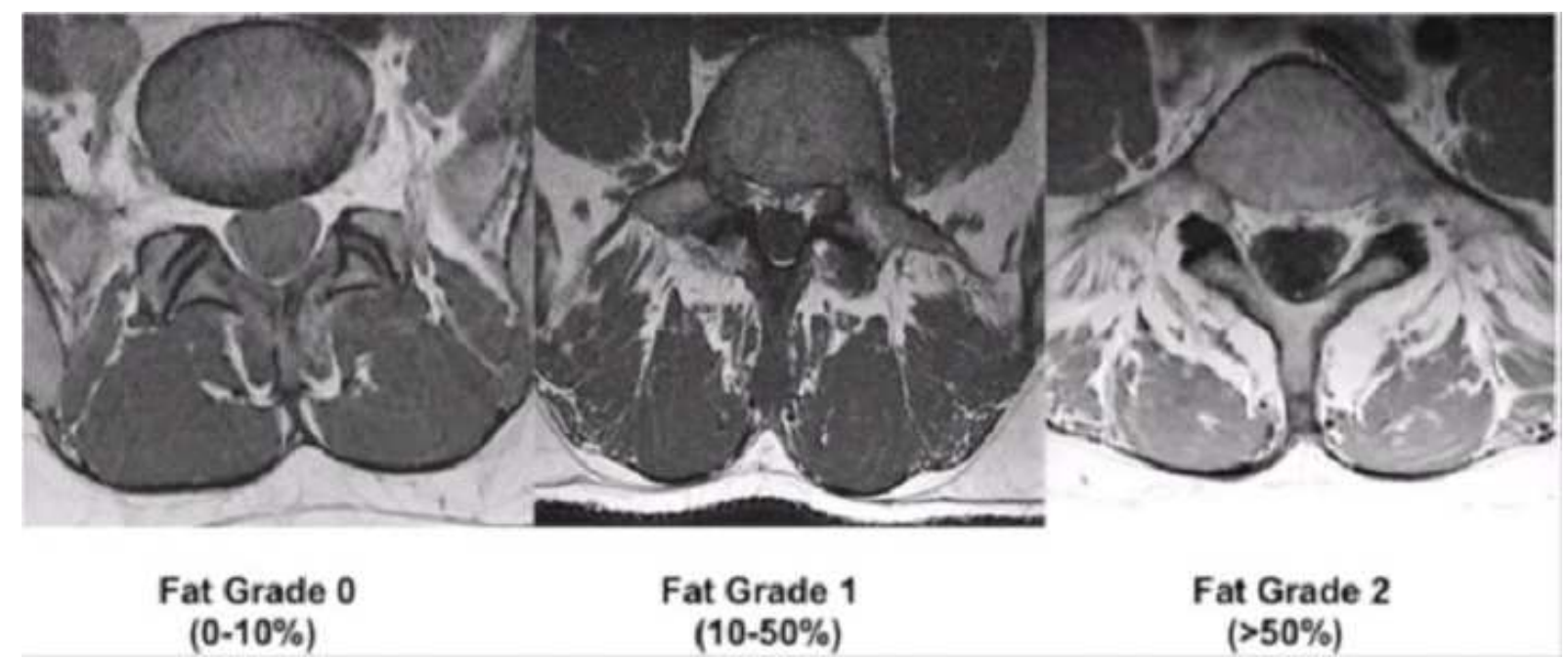

Fig. 1. Grading of MR images with different muscle-fat components of the lumbar multifidus muscle. 


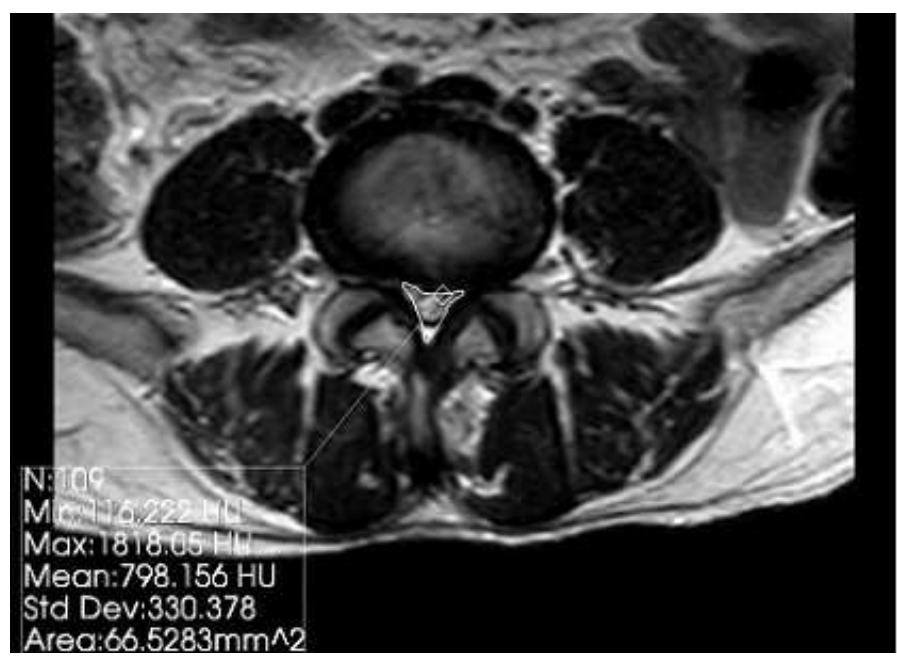

Fig. 2. SCCSA measurement at L4-5 level.

of the control group was $58.34 \pm 7.7$ years. The age range of the study group was 39-75 years and that of the control group was 39-76. SCCSA at the stenosis level (mm2), PSCSA (mm2) at the L3 level, MFCSA (mm2) at the L3-L4 and the L4-L5 levels, and the degrees of multifidus muscle atrophy were evaluated using MRI and compared with the control group. Adana City Training and Research Hospital Clinical Research Ethics Committee approved the study. Registration number: 1202 , registration date: $30 / 12 / 2020$.

\section{Imaging Parameters}

An MRI of the lumbar spine was performed using a 1.5-T unit (Philip, Ingenia; Philips Medical Systems, Best, the Netherlands). The imaging protocols included axial T2-weighted images (TR/TE 2770/100, matrix size $200 \times 138$, slice thickness $4 \mathrm{~mm}$ ) and sagittal T2-weighted images (TR/ TE 3403/100, matrix size $160 \times 232$, slice thickness $4 \mathrm{~mm}$ ).

\section{Image Analysis}

The lumbar MR images were evaluated by an experienced neuroradiologist, blinded to the symptoms, using a digital workstation. The measurements were done by one radiologist who repeated the measurements three times on different days to reduce the possibility of a bias. First, T2-weighted sagittal and axial images were used to determine the level and the crosssectional area of the stenosis. The severity of multifidus fatty degeneration was graded according to the fat infiltration grading system.

T2-weighted axial MR images were used for the evaluation of the multifidus muscles and psoas muscles. MFCSA, PSCSA, and SCCSA at the stenosis level were examined to assess muscle atrophy. The SCCSA was measured on the axial T2-weighted MR images at the level of the stenosis, and the PSCSA at the L3 level. SCCSA was measured by measuring the area between the posterior edge of the posterior longitudinal ligament and the inner edge of the ligamentum flavum (Fig. 2). The MFCSA (Fig. 3) and PSCSA (Fig. 4) were traced using the manual cursor technique. The MFCSA and the PSCSA were measured on the right side of the spine.

\section{Statistical Analysis}

In the study, the demographic and clinical characteristics of the participants in the patient and control

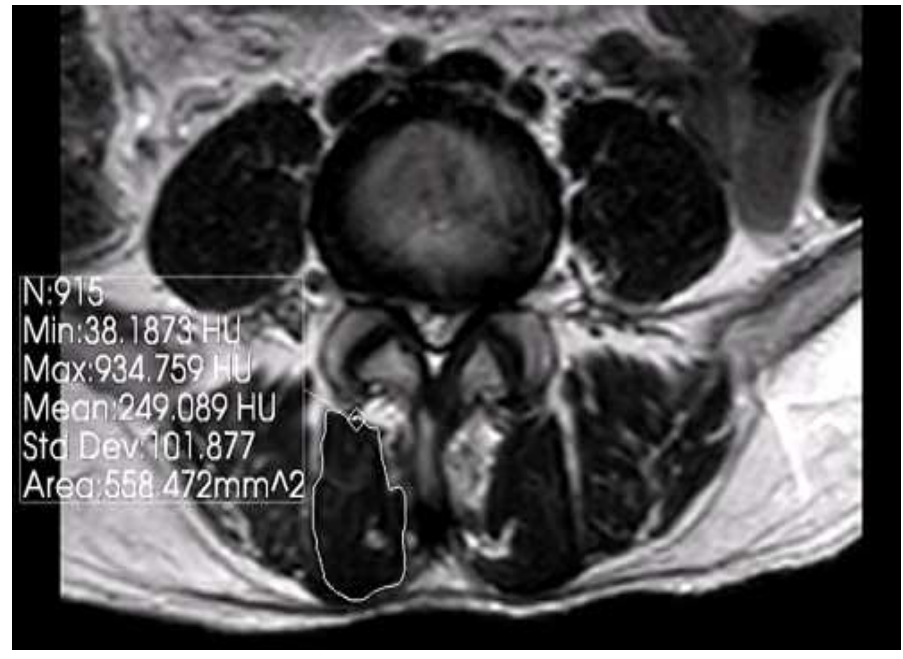

Fig. 3. MFCSA measurement at L4-5 level.

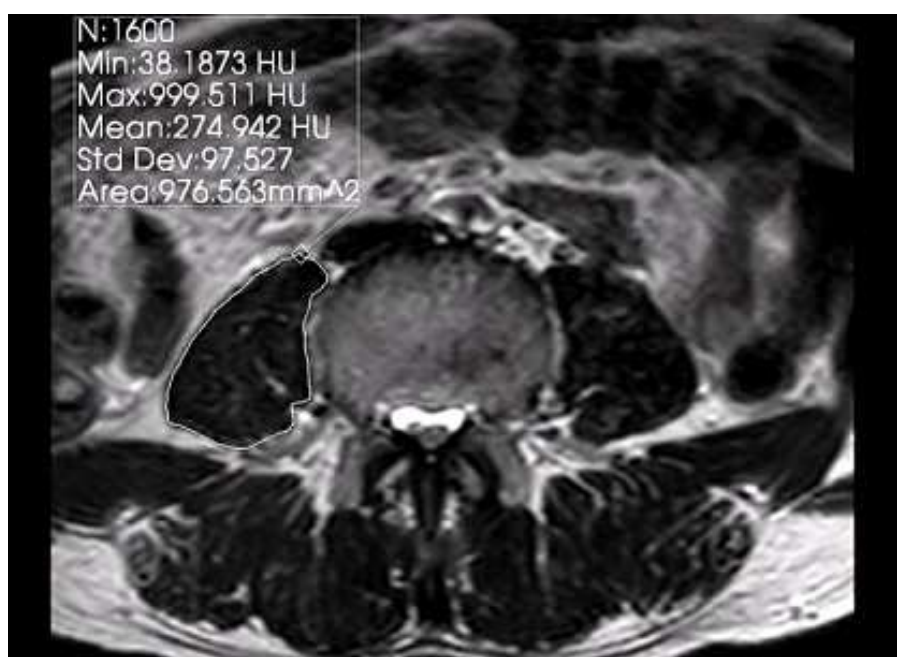

Fig. 4. PSCSA measurement at L3 level. 
groups were evaluated with descriptive statistical analysis regarding the number, percentage, mean, standard deviation, and median values. The degree of multifidus fatty degeneration among the participants in the patient group and the control group was evaluated using Fisher's exact test. In addition, gender ratios between the patient and control groups were evaluated according to the Pearson chi-squared analysis. The mean L3 PSCSA between the two groups was determined by the Mann-Whitney U test; MFCSA at the L3-4 level, MFCSA at the L4-5 level, MFCSA at the L5-S1 level, the L3-L4 spinal blood CSA, and the L4L5 spinal blood CSA were compared with an Independent Groups $t$ test. Among the men and women in both the patient group and the control group; PSCSA at the L3 level, MFCSA at the L3-4 level, MFCSA at the L4-5 level, MFCSA at the L5-S1 level, L3-L4 SCCSA and the L4-L5 SCCSA averages were compared using the Mann-Whitney U test. PSCSA at the L3 level, MFCSA at the L3-4 level, MFCSA at the L45 level, MFCSA at the L5-S1 level, the L3-L4 SCCSA and the L4-L5 SCCSA averages were compared using the Independent Groups $t$ test. PSCSA at the L3 level, MFCSA at the L3-4 level, MFCSA at the L4-5 level, MFCSA at the L5-S1 level, the L3-L4 SCCSA, and the L4-L5 SCCSA relationships were analyzed using the Pearson's correlation analysis for normally distributed data, and the Spearman's correlation analysis for non-normally distributed data. The normal distribution compliance of the Independent Groups $t$ test and the Pearson's correlation analysis were checked with kurtosis and skewness values $( \pm 1.5)$. The significance level for all analyses was set as $p<0.05$. The IBM
SPSS 22.0 program application was used for the analysis.

\section{RESULTS}

The mean age of the patients evaluated was 58.20 \pm 8.34 years (min. 39.00 ; $\max .75 .00$ ), and the mean age of the participants in the control group was 58.34 \pm 7.70 (min. 39.00; max. 76.00). According to the $t$ test, the mean age of the two groups were statistically similar $(p=0.916)$. There were $54(38.6 \%)$ men in the patient group and the control group and according to the Pearson chi-squared analysis, it was found that the gender ratio between the two groups was $\operatorname{similar}(p=$ 0.999).

In the patient group, $60(42.9 \%)$ had multifidus muscle fatty atrophy grade one, $76(54.3 \%)$ had fatty atrophy grade two, and four (2.9\%) had fatty atrophy grade three. The control group was found to have 116 $(82.9 \%)$ of the participants with multifidus muscle fatty atrophy grade one, and $24(17.1 \%)$ had fatty atrophy grade two. According to the Fisher exact test results, it was found that the degree of multifidus muscle fatty atrophy between the patient and control groups was statistically significantly different.

According to the Mann-Whitney $U$ test, it was found that the mean PSCSA at the L3 level in the patient group was not statistically significantly different from the control group ( $p=0.067)$. According to the Independent Groups t test, the MFCSA at the L3-4 level ( $p<0.001)$, the MFCSA at the L4-5 level ( $p<$ $0.001)$, the MFCSA at the L5-S1 level $(p<0.001)$, L3-

Table 1. Comparison of the averages of L3 psoas area, MFCSA L3-4, MFCSA L4-5, MFCSA L5S1, L3-L4 SCCSA and L4-L5 SCCSA between patient (PG) and control groups (CG)

\begin{tabular}{|c|c|c|c|c|c|c|c|}
\hline & \multicolumn{3}{|c|}{ PG } & \multicolumn{3}{|c|}{ CG } & \multirow[t]{2}{*}{$p$ value } \\
\hline & mean & SD./med & $\min -\max$ & mean & SD./med. & $\min -m a x$ & \\
\hline L3 psoas area & 816.02 & 798.93 & $400.98-1421.41$ & 917.46 & 832.50 & $484.01-2006.54$ & $0.067^{\mathrm{a}}$ \\
\hline MF L3-4 & 431.10 & 80.11 & 260.79-609.05 & 535.83 & 102.78 & $361.92-879.31$ & $<\mathbf{0 . 0 0 1}^{\mathrm{b}}$ \\
\hline MF L4-5 & 574.71 & 105.32 & $370.69-818.35$ & 719.97 & 115.59 & $490.14-976.84$ & $<0.001^{\mathrm{b}}$ \\
\hline MF L5-S1 & 666.32 & 133.73 & $410.19-1011.64$ & 820.06 & 132.52 & $514.20-1157.53$ & $<\mathbf{0 . 0 0 1}^{\mathrm{b}}$ \\
\hline L3-L4 & 91.77 & 18.40 & $57.39-124.36$ & 208.48 & 36.48 & $149.71-292.26$ & $<0.001^{\mathrm{b}}$ \\
\hline L4-L5 & 97.52 & 21.81 & $49.43-136.32$ & 201.29 & 39.22 & $146.04-286.68$ & $<0.001^{\text {b }}$ \\
\hline
\end{tabular}

MFCSA $=$ cross-sectional areas of the multifidus, SCCSA $=$ spinal canal cross-sectional area, med. = median, min. $=$ minimum, max. $=$ maximum, ${ }^{\mathrm{a}}$ Mann-Whitney $\mathrm{U}$ test, ${ }^{\mathrm{b}}$ Independent Groups $\mathrm{t}$ test 
L4 SCCSA $(p<0.001)$ and the L4-L5 SCCSA $(p<$ 0.001 ) were found to be statistically significantly lower than the mean of the cases in the control group (Table 1).

According to the Mann-Whitney U test, the means of the PSCSA at the L3 level $(p<0.001)$, MFCSA at the L3-4 level $(p<0.001)$, MFCSA at the L4-5 level $(p<0.001)$, and the MFCSA at the L5-S1 level ( $p=$ 0.001 ) was found to be statistically significantly higher in the men than the means of the women in the patient group. According to the Mann-Whitney $U$ test, the means of the PSCSA at the L3 level $(p<0.001)$, the MFCSA at the L3-4 level $(p<0.001)$, the MFCSA at the L4-5 level $(p<0.001)$, and the MFCSA at the L5S1 level $(p<0.001)$ for the men in the control group was found to be statistically significantly higher than the means of women. According to the Mann-Whitney $\mathrm{U}$ test, the means of the MFCSA at the L3-4 level ( $p$ $<0.001)$, MFCSA at the L4-5 level $(p<0.001)$, and MFCSA at the L5-S1 level $(p=0.001)$, the L3-L4 $\operatorname{SCCSA}(p<0.001)$ and the L4-L5 SCCSA $(p<0.001)$ for the men in the patient group was found to be statistically significantly lower than the means for the men in the control group. According to the Independent Groups t test of the women in the patient group the means of the multifidus psoas muscle CSA at the L3 ( $p=0.043)$, the MFCSA at the L3-4 level $(p<0.001)$, MFCSA at the L4-5 level ( $p<0.001)$, MFCSA at the L5-S1 level $(p<0.001)$, and the L4-L5 SCCSA ( $p<$ 0.001 ) was found to be statistically significantly lower than the means of women in the control group. In addition, according to the Mann-Whitney U test, the L3L4 level of the women in the patient group was found to be statistically significantly lower $(p<0.001)$ than the mean SCCSA of women in the control group (Table 2).

According to the Spearman correlation analysis, PSCSA values at the L3 level and the MFCSA at the L3-4 level $(\mathrm{r}=0.555, p<0.01)$, the MFCSA at the L45 level $(\mathrm{r}=0.559, p<0.01)$, and the MFCSA at the L5-S1 level $(\mathrm{r}=0.429, p<0.01)$, were found to have a statistically significant positive correlation. According to the Pearson correlation analysis, the L4-L5 level of the cases in the control group was found to have a statistically significant positive relationship between the SCCSA values and the MFCSA at the L3-4 level $(\mathrm{r}=0.343, p<0.01)$, the MFCSA at the L4-5 level ( $\mathrm{r}$ $=0.404, p<0.01)$, and the MFCSA at the L5-S1 level

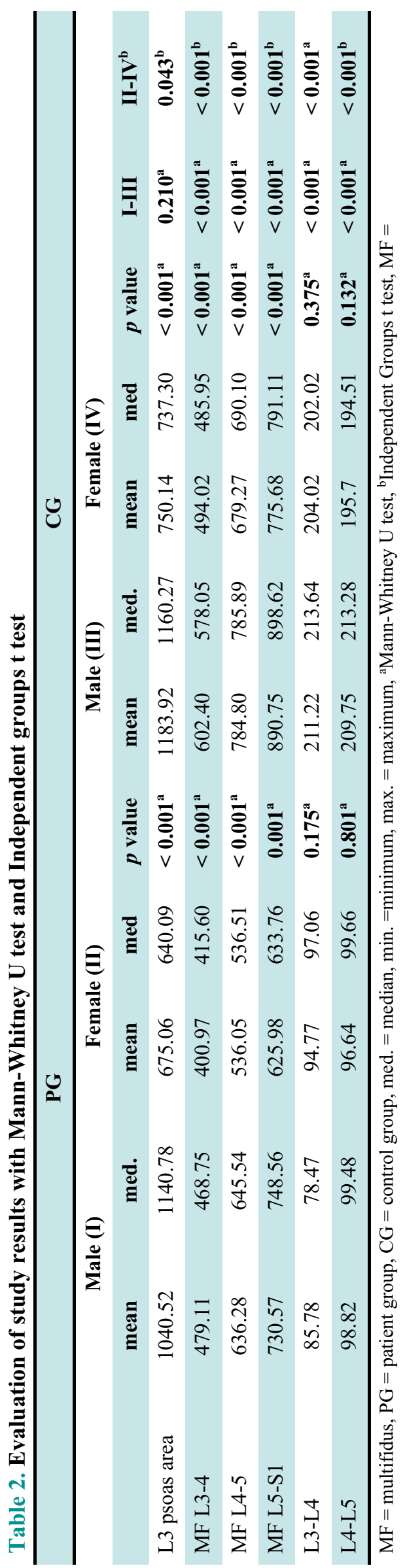




\begin{tabular}{|c|c|c|c|c|c|c|c|}
\hline Group & & $\mathbf{L} 3-\mathbf{L} 4^{\mathrm{a}}$ & $\mathbf{L}^{4}-\mathbf{L 5}^{\mathbf{a}}$ & $\begin{array}{l}\text { L3 psoas } \\
\text { area }^{\text {b }}\end{array}$ & MF L3-4 ${ }^{\text {a }}$ & MF L4-5 ${ }^{\mathrm{a}}$ & MF L5-S1 ${ }^{\text {a }}$ \\
\hline \multirow[t]{6}{*}{ PG } & $\mathrm{L} 3-\mathrm{L} 4^{\mathrm{a}}$ & 1.000 & & & & & \\
\hline & L4-L5 ${ }^{\mathrm{a}}$ & 0.357 & 1.000 & & & & \\
\hline & L3 psoas areab & -0.080 & -0.026 & 1.000 & & & \\
\hline & MF L3-L4 ${ }^{\mathrm{a}}$ & 0.013 & 0.121 & $0.555^{* *}$ & 1.000 & & \\
\hline & MF L4-L5 ${ }^{\mathrm{a}}$ & 0.108 & 0.122 & $0.559^{* *}$ & $0.810^{* *}$ & 1.000 & \\
\hline & MF L5-S1 ${ }^{\mathrm{a}}$ & -0.237 & 0.114 & $0.429^{* *}$ & $0.715^{* *}$ & $0.840^{* *}$ & 1.000 \\
\hline \multirow[t]{6}{*}{$\mathrm{CG}$} & $\mathrm{L} 3-\mathrm{L}^{\mathrm{a}}$ & 1.000 & & & & & \\
\hline & $\mathrm{L} 4-\mathrm{L}^{\mathrm{a}}$ & $0.856^{* *}$ & 1.000 & & & & \\
\hline & L3 psoas area ${ }^{b}$ & 0.039 & 0.123 & 1.000 & & & \\
\hline & MF L3-L4 ${ }^{\mathrm{a}}$ & 0.197 & $0.343^{* *}$ & $0.622^{* *}$ & 1.000 & & \\
\hline & MF L4-L5 ${ }^{\mathrm{a}}$ & $0.264^{*}$ & $0.404^{* *}$ & $0.470^{* *}$ & $0.795^{* *}$ & 1.000 & \\
\hline & MF L5-S1 ${ }^{\mathrm{a}}$ & 0.190 & $0.336^{* *}$ & $0.468^{* *}$ & $0.777^{* *}$ & $0.865^{* *}$ & 1.000 \\
\hline
\end{tabular}

$\mathrm{MF}=$ multifidus, $\mathrm{PG}=$ patient group, $\mathrm{CG}=$ control group, ${ }^{\mathrm{a}}$ Pearson Correlation Analysis, ${ }^{\mathrm{b}}$ Spearman Correlation Analysis, $* *<0.001 . *<0.01$

$(\mathrm{r}=0.336, p<0.01)$. According to the Spearman correlation analysis, a statistically significant positive relationship was found between the PSCSA values at the L3 level and the MFCSA at the L3-4 level $(r=0.622$, $p<0.01)$, the MFCSA at the L4-5 level $(\mathrm{r}=0.470, p$ $<0.01)$, and the MFCSA at the L5-S1 level $(\mathrm{r}=0.468$, $p<0.01$ ) (Table 3).

\section{DISCUSSION}

Lumbar spinal stenosis is a common disease. The occurrence rate of this disease has increased significantly in recent years due to the increase in life expectancy. The introduction of new diagnostic methods in clinical practice has brought new recommendations for radiological criteria in spinal canal evaluation. MRI has become the preferred diagnostic tool for lumbar spinal stenosis because it can visualize muscle and other soft tissues. Evaluation of multifidus muscle atrophy in a diagnostic lumbar MRI will be advantageous in guiding the treatment process.

A limited number of studies have examined changes in lumbar paraspinal muscle cross-sectional areas. None of these studies have compared PSCSA, MFCSA, and SCCSA at the spinal stenosis level in patients with lumbar spinal stenosis. Takayama et al.
[13] studied the cross-sectional area of the paraspinal muscles using T2-weighted MRI in 160 patients aged between $10-88$ years. They showed that the cross-sectional area of the paraspinal muscles tends to decrease with age. Sasaki et al. [14] achieved the same result in their studies. In our study, we found that the crosssectional area of the paraspinal muscles decreases with increasing age in agreement with the literature. We anticipate that exercise may prevent narrow canal development caused by age-related multifidus muscle and psoas muscle atrophy.

In addition, men are known to have a larger paraspinal muscle cross section than women [13], and in our analysis, we found a statistically significant difference in that the cross-sectional area of the psoas muscle and multifidus muscle in men is higher than in women.

Zhao et al. [15] and Yoshihara et al. [16] reported histological studies showing significant atrophy of the multifidus muscles in patients with lumbar disc herniation. Hodges et al. [17] found rapid atrophy of the multifidus muscles after root compression in his experimental studies. In our study, we found statistically significant atrophy due to fatty degeneration in multifidus muscles due to root compression in patients with spinal stenosis with narrowed spinal canal incisional area similar to lumbar disc herniation. At the same 
time, we found that there was no statistically significant difference in the PSCSA at the L3 level in patients with spinal stenosis compared to the control group. In our study, it was found that atrophy and fatty degeneration of the multifidus muscle due to compression of the spinal root at the spinal canal level were statistically significantly associated, but the PSCSA did not change at the L3 level in patients with spinal canal stenosis. The diagnostic evaluation of patients with lumbar MRI, together with an indication of the degree of multifidus muscle fatty degeneration atrophy should be considered in terms of stenosis prognosis and treatment. However, additional studies will be required to evaluate the overall paraspinal muscle crosssectional area, muscle density, effects on spinal stability, and changes before and after surgery.

\section{Limitations}

The limitations of our study were that the BMI index of the patients was not known, the measurement parameters that would evaluate lumbar stability other than the cross-sectional muscle areas of the patients were not evaluated, and the cross-sectional area comparison before and after the surgery was not performed.

\section{CONCLUSION}

The study found that atrophy and fatty degeneration of the multifidus muscle due to compression of the spinal root at the spinal canal level were statistically significantly associated, but the cross-sectional area of the psoas muscle did not change at the L3 level in patients with spinal canal stenosis.

\section{Authors' Contribution}

Study Conception: GÇ; Study Design: GÇ; Supervision: GÇ; Funding: YÇ; Materials: YÇ; Data Collection and/or Processing: YÇ; Statistical Analysis and/or Data Interpretation: GÇ; Literature Review: GÇ; Manuscript Preparation: GÇ and Critical Review: YÇ.

\section{Conflict of interest}

The authors disclosed no conflict of interest during the preparation or publication of this manuscript.

\section{Financing}

The authors disclosed that they did not receive any grant during conduction or writing of this study.

\section{REFERENCES}

1. Sullivan MS. Back support mechanisms during manual lifting. Phys Ther 1989;69:38-45.

2. Kader DF, Wardlaw D, Smith FW. Correlation between the MRI changes in the lumbar multifidus muscles and leg pain. Clin Radiol 2000;55:145-9.

3. Farshad M, Gerber C, Farshad-Amacker NA, Dietrich TJ, Laufer-Molnar V, Min K. Asymmetry of the multifidus muscle in lumbar radicular nerve compression. Skeletal Radiol 2014;43:49-53.

4. Boissière L, Moal B, Gille O, De-Roquefeuil E, Durieux M, Obeid I, et al. Lumbar spinal muscles and spinal canal study by MRI three-dimensional reconstruction in adult lumbar spinal stenosis. Orthop Traumatol Surg Res 2017;103:279-83.

5. Saifuddin A. The imaging of lumbar spinal stenosis. Clin Radiol 2000;55:581-94.

6. An SJ, Mun JU, Kang KN, Kim YU. Superior articular process cross-sectional area is a new sensitive parameter for the diagnosis of lumbar central canal spinal stenosis. Clin Interv Aging 2018;13:1763-7.

7. Hicks GE, Simonsick EM, Harris TB, Newman AB, Weiner DK, Nevitt MA, et al. Cross-sectional associations between trunk muscle composition, back pain, and physical function in the health, aging and body composition study. J Gerontol A Biol Sci Med Sci 2005;60:882-7.

8. Kamath S, Venkatanarasimha N, Walsh MA, Hughes PM. MRI appearance of muscle denervation. Skeletal Radiol 2008;37:397404.

9. Hildebrandt M, Fankhauser G, Meichtry A, Luomajoki H. Correlation between lumbar dysfunction and fat infiltration in lumbar multifidus muscles in patients with low back pain. BMC Musculoskelet Disord 2017;18:1-9.

10. Barker KL, Shamley DR, Jackson D. Changes in the crosssectional area of multifidus and psoas in patients with unilateral back pain: the relationship to pain and disability. Spine (Phila $\mathrm{Pa}$ 1976) 2004;29:515-9.

11. De Sèze MP, Guérin P, Gille O, Jolivet E, Skalli W. Reliability of magnetic resonance imaging measurements of the cross-sectional area of the muscle contractile and non-contractile components. Surg Radiol Anat 2011;33:735-41.

12. Yagi M, Hosogane N, Watanabe K, Asazuma T, Matsumoto M. The paravertebral muscle and psoas for the maintenance of global spinal alignment in patient with degenerative lumbar scoliosis. Spine J 2016;16:451-8.

13. Takayama K, Kita T, Nakamura H, Kanematsu F, Yasunami $\mathrm{T}$, Sakanaka H, et al. New predictive index for lumbar paraspinal muscle degeneration associated with aging. Spine (Phila Pa 1976) 2016;41:E84-E90.

14. Sasaki T, Yoshimura N, Hashizume H, Yamada H, Oka H, 
Matsudaira K, et al. MRI-defined paraspinal muscle morphology in Japanese population: The Wakayama Spine Study. PLoS One 2017;12:1-15.

15. Zhao WP, Kawaguchi Y, Matsui H, Kanamori M, Kimura T. Histochemistry and morphology of the multifidus muscle in lumbar disc herniation: Comparative study between diseased and normal sides. Spine (Phila Pa 1976) 2000;25:2191-9.
16. Yoshihara K, Shirai Y, Nakayama Y, Uesaka S. Histochemical changes in the multifidus muscle in patients with lumbar intervertebral disc herniation. Spine (Phila Pa 1976) 2001;26:622-6. 17. Hodges P, Holm AK, Hansson T, Holm S. Rapid atrophy of the lumbar multifidus follows experimental disc or nerve root injury. Spine (Phila Pa 1976) 2006;31:2926-33. 